\title{
Regulation of the catalytic behaviour of L-form starch phosphorylase from sweet potato roots by proteolysis
}

\author{
Han-Min Chen, Shih-Chung Chang, Chi-Chen Wu, Ting-Shen Cuo, Jiann-Shing Wu and Rong-Huay Juang* \\ Department of Agricultural Chemistry, National Taiwan University, Taipei, Taiwan 106 \\ *Corresponding author,e-mail: juang@ccms.ntu.edu.tw
}

Received 28 May 2001; revised 14 November 2001

Starch phosphorylase (SP) is an enzyme used for the reversible phosphorolysis of the $\alpha$-glucan in plant cells. When compared to its isoform in an animal cell, glycogen phosphorylase, a peptide containing 78 amino acids (L78) is inserted in the centre of the low-affinity type starch phosphorylase (LSP). We found that the amino acid sequence of $\mathrm{L} 78 \mathrm{had}$ several interesting features including the presence of a PEST region, which serves as a signal for rapid degradation. Indeed, most L-SP molecules isolated from mature sweet potato roots were nicked in the middle of a molecule, but still retained their tertiary or quaternary structures, as well as full catalytic activity. The nicking sites on the $\mathrm{L} 78$ were identified by amino acid sequencing of these peptides, which also enabled us to propose a proteolytic process for L-SP. Enzyme kinetic studies of L-SP in the direction of starch synthesis indicated that the $K_{\mathrm{m}}$ decreased during the proteolytic process when starch was used as the limiting substrate, but the $K_{m}$ for the other substrate (Glc-1-P) increased. On the other hand, the maximum velocities $\left(V_{\max }\right)$ increased for both substrates. Mobility of the nicked L-SP was retarded on a native polyacrylamide gel containing soluble starch, indicating the increased affinity for starch. Results in this study suggested that L78 and its proteolytic modifications might play a regulatory role on the catalytic behaviour of L-SP in starch biosynthesis.

\section{Introduction}

Starch phosphorylase (SP, EC 2.4.1.1) catalyses the reversible phosphorolysis of $\alpha$-glucan, and produces glucose-1-phosphate (Glc-1-P) as one of the products (Hanes 1940). Tsai and Nelson (1968) identified two major types of SP having distinctive catalytic behaviour in the endosperm of Zea mays. In their study, one of the SP isoforms (phosphorylase II) appeared only at the stage of rapid starch biosynthesis and was not found during germination. The isoforms of phosphorylase from potato tubers are classified as high-affinity type (H-SP, or type II SP) and low-affinity type (L-SP, or type I SP) according to their affinities to starch (Nakano et al. 1989). When compared to the amino acid sequence of glycogen phosphorylase, a 78-amino acid insertion (L78) is presented in the middle of the L-SP isoform (Nakano and Fukui 1986). In addition, Camirand et al. (1990) suggested that the DNA sequence coding for L78 might be derived from an intron. This L78 is inserted in the starch-binding region of L-SP, its helical structure ex- tends outwards from the molecule, and exposes itself into the hydrophilic environment. Consequently, L78 hinders the binding of L-SP to starch, possibly decreasing the affinity of L-SP for starch (Mori et al. 1993a, 1993b). Similar to the potato phosphorylase, the L-SP from sweet potato consists of two identical subunits (110 $\mathrm{kDa}$ ) and has a native molecular mass of $220 \mathrm{kDa}$. Lin et al. (1991) deduced the primary structure of sweet potato L-SP from its cDNA sequence. Our previous study indicated that the sweet potato L-SP molecule was susceptible to degradation and produced fragments having the molecular mass around $50 \mathrm{kDa}$ (F50) when examined on denaturing polyacrylamide gels (Chiang et al. 1991). According to those results, L-SP was nicked at several sites on the L78. Although most L-SP molecules isolated from sweet potato roots were nicked, the catalytic activity appeared to persist (Chern et al. 1990).

Considerable attention has been paid to complex regulatory mechanisms that control glycogen phosphorylase

Abbreviations - Glc-1-P, glucose-1-phosphate; H-SP, high-affinity type starch phosphorylase; L-SP, low-affinity type starch phosphorylase; PBST, phosphate buffered saline containing Tween; SP, starch phosphorylase. 
such as protein phosphorylation and allosteric regulation (Newgard et al. 1989, Johnson and Barford 1990). None of these regulatory properties had been found in starch phosphorylase (Nakano and Fukui 1986). Nevertheless, Brisson et al. (1989) proposed a possible control mechanism of SP by the compartmentation inside or outside the amyloplast. Owing to the fact that SP is one of the important enzymes in the carbohydrate metabolism of a higher plant (Tsai et al. 1970), the existence of a regulatory mechanism might be needed for an efficient control of its activity. The L78 insertion in the starch-binding site of L-SP, and the subsequent proteolytic degradation of this peptide, might serve as a regulatory mechanism for the enzyme in plants. In this study, we examine the unique structural features on the L78 sequence, analyse the proteolytic process of L78, and observe the increase of substrate affinity and catalytic velocity of L-SP during the proteolytic modification. Results in this study point towards a possible role for L-SP in starch metabolism.

\section{Materials and methods}

\section{Materials}

Mature roots of sweet potato (Ipomoea batatas [L.] Lam. cv. Tainong 57) were collected from a local farm near Taipei. The roots were stored in the cold place and used for the analysis or purification procedures.

\section{Assays for protein content and phosphorylase activity}

Protein content was determined by the dye-binding method (Bradford 1976) using the microassay system from Bio-Rad (Protein Assay Kit). Bovine serum albumin was used as the standard.

SP activity in the direction of starch synthesis was assayed in microtiter plates. The reaction mixture contained $50 \mathrm{~m} M$ sodium acetate, $\mathrm{pH} 5.4,0.3 \%(\mathrm{w} / \mathrm{v})$ potato soluble starch (Sigma S-2630), $8 \mathrm{~m} M$ potassium Glc-1$\mathrm{P}$, and the enzyme sample in a final volume of $100 \mu \mathrm{l}$. After incubation at $37^{\circ} \mathrm{C}$ for $10 \mathrm{~min}$, the inorganic phosphate released was determined by adding $200 \mu \mathrm{l}$ of ferrous sulphate molybdate solution to each well, according to the procedure of Fiske and Subbarow (1925). After $10 \mathrm{~min}$ of incubation at room temperature, the absorbance was measured by an ELISA reader at 650 $\mathrm{nm}$.

\section{Purification of L-form starch phosphorylase}

Sweet potato SP was purified according to a method described elsewhere (Chang et al. 1987) with slight modifications. All purification procedures were carried out at $0-4^{\circ} \mathrm{C}$ using buffer A $(50 \mathrm{~m} M$ imidazole, $\mathrm{pH} 7.0$, containing $0.1 \mathrm{~m} M$ EDTA, and $0.2 \mathrm{M} \mathrm{NaCl}$ ) as the buffer system. Fresh sweet potato roots $(300 \mathrm{~g})$ were added to $300 \mathrm{ml}$ of extraction buffer (buffer A containing 1\% PVP), and were homogenized in a Waring blender. The crude extract was collected by centrifugation (10 $000 \mathrm{~g}$, $40 \mathrm{~min}$ ) and was precipitated by adding solid ammonium sulphate to $80 \%$ saturation at $0^{\circ} \mathrm{C}$. The protein was then collected by centrifugation $(10000 \mathrm{~g}, 40 \mathrm{~min})$ and resuspended in a minimal amount of buffer $\mathrm{A}$. The light brownish mixture was dialysed overnight against buffer $\mathrm{A}$, and the precipitate subsequently formed was removed by centrifugation (10 $000 \mathrm{~g}, 40 \mathrm{~min})$ before applying it to a DEAE-Sephacel column (i.d. $2.6 \times 20 \mathrm{~cm}$, pre-equilibrated in buffer A). After washing with one or two volumes with buffer $\mathrm{A}$, the column was eluted with a linear gradient of $\mathrm{NaCl}(0.2-0.5 \mathrm{M})$. Fractions with enzyme activity were collected and concentrated by ultrafiltration (Amicon YM-10). It was further purified by gel filtration using Sephacryl S-300 (i.d. $2.6 \times 100 \mathrm{~cm}$, equilibrated in buffer $\mathrm{A}$ ). The activity fractions eluted from the column were combined and concentrated as above. SP was then subjected to preparative gel electrophoresis (7.5\% disc-PAGE) as described elsewhere (Juang and $\mathrm{Su}$ 1988). The SP band was detected by activity staining of sampling strips, which were sliced from both lateral sides of the gel. The band was cut out and then the enzyme was eluted from the gel by an electrophoretic concentrator (Little Blue Tank and ISCO 1750). This protocol allowed us to obtain an electrophoretically pure enzyme, which was used in all experiments of this study.

\section{Antibody production}

Monoclonal antibodies against sweet potato SP were prepared according to the procedure described earlier (Chern et al. 1990). BALB/c mouse was immunized by injecting $0.3 \mathrm{ml}$ of antigen emulsion containing $100 \mu \mathrm{g}$ $\mathrm{ml}^{-1}$ of purified SP in complete Freund's adjuvant. Three or four booster shots were taken every 2 weeks in which the incomplete adjuvant was used instead. Cell fusion was initiated by mixing $2 \times 10^{7} \mathrm{NS}-1$ myeloma cells to $10^{8}$ spleen cells from the immunized mouse. The cells were collected by centrifugation at $150 \mathrm{~g}$ for $5 \mathrm{~min}$. Next, $0.7 \mathrm{ml}$ of PEG 1500 (50\%, Böehringer Mannheim 783 641) was slowly mixed to the resuspended cell for a period of $2 \mathrm{~min}$. The PEG in the cell mixture was diluted immediately by adding $10 \mathrm{ml}$ of RPMI-1640 in a drop wise manner for $5 \mathrm{~min}$ and then centrifuged at $120 \mathrm{~g}$ for $10 \mathrm{~min}$. The pellet was resuspended gently in $30 \mathrm{ml}$ of RPMI-1640 containing 15\% foetal calf serum and HAT (final concentration in $100 \mathrm{ml}: 1.36 \mathrm{mg}$ hypoxanthine, $17.6 \mu \mathrm{g}$ aminopterin, and $0.39 \mathrm{mg}$ thymidine) which served as an initial screening for fused cells. The cell suspension was incubated in the $\mathrm{CO}_{2}$ incubator for $1 \mathrm{~h}$ before it was evenly distributed into three 96-well microtiter plates. For the next 3 days, one drop of RPMI-1640 containing 10\% fetal calf serum and HT (hypoxanthine and thymidine as above) was added to each well daily. Clones producing antibodies specific to SP were further screened by ELISA and subcloned by the limiting dilution method. Monoclonal antibodies were produced by inducing ascites fluid in BALB/c mice and, then, the immunoglobulin fraction was isolated by 
ammonium sulphate fractionation (40\% saturation). Two cell lines producing monoclonal antibodies ( $\mathrm{J} 3 \mathrm{~b}$ and H7c) specific to distinct epitopes on L-SP (Chern et al. 1990) were used in this study.

Synthetic peptides (L1 and L2, see Fig. 1C for their amino acid sequences) were obtained from a commercial service provided by Chiron Technologies (Australia). For the selected amino acid fragment, a Cys residue was added to the $\mathrm{C}$-terminus. The Cys was used to conjugate the peptide with the maleimide activated keyhole lympet haemocyanin. The conjugates were then used to immunize mice, and the ascites fluid was induced by injecting NS-1 myeloma cell intraperitoneally as described elsewhere (Harlow and Lane 1988).

\section{Polyacrylamide gel electrophoresis, protein transfer and immunostaining}

SDS-PAGE was performed according to the method of Laemmli (Laemmli 1970) with slight modifications using a mini-slab gel system (Hoefer SE250). After electrophoresis, protein bands were stained by Coomassie Brilliant Blue or ammoniacal silver. Otherwise, the gel was transferred to the nylon paper (Immobilon-P, Millipore) in a Mighty Small Transphor (Hoefer TE22) at $0.5 \mathrm{~A}$ for $1 \mathrm{~h}$ in the transfer buffer $(25 \mathrm{~m} M$ Tris- $\mathrm{HCl}, \mathrm{pH} 8.3$, and $0.192 M$ glycine containing $10 \%$ methanol). The protein blot was washed overnight with three changes of $6 M$ urea in PBST (phosphate buffered saline containing $0.05 \%$ Tween) to remove SDS and partially renature protein for immunostaining. The washed blot was immersed in a solution of antibody diluted properly in gelatinNET $(0.25 \%$ gelatin, $0.15 \mathrm{M} \mathrm{NaCl}, 5 \mathrm{~m} M$ EDTA, $0.05 \%$ Tween in $50 \mathrm{~m} M$ Tris- $\mathrm{HCl}, \mathrm{pH} 8.0$ ), and was incubated at room temperature for $1 \mathrm{~h}$. The blot was then washed three times with PBST. The biotinylated second antibody (goat antimouse $\operatorname{IgG}, \operatorname{IgA}$ and $\operatorname{IgM}$ ) diluted in gelatin-NET $(1: 2000)$ was added. That antibody was incubated and washed as mentioned earlier. Next, the streptavidin-biotinylated alkaline phosphatase complex

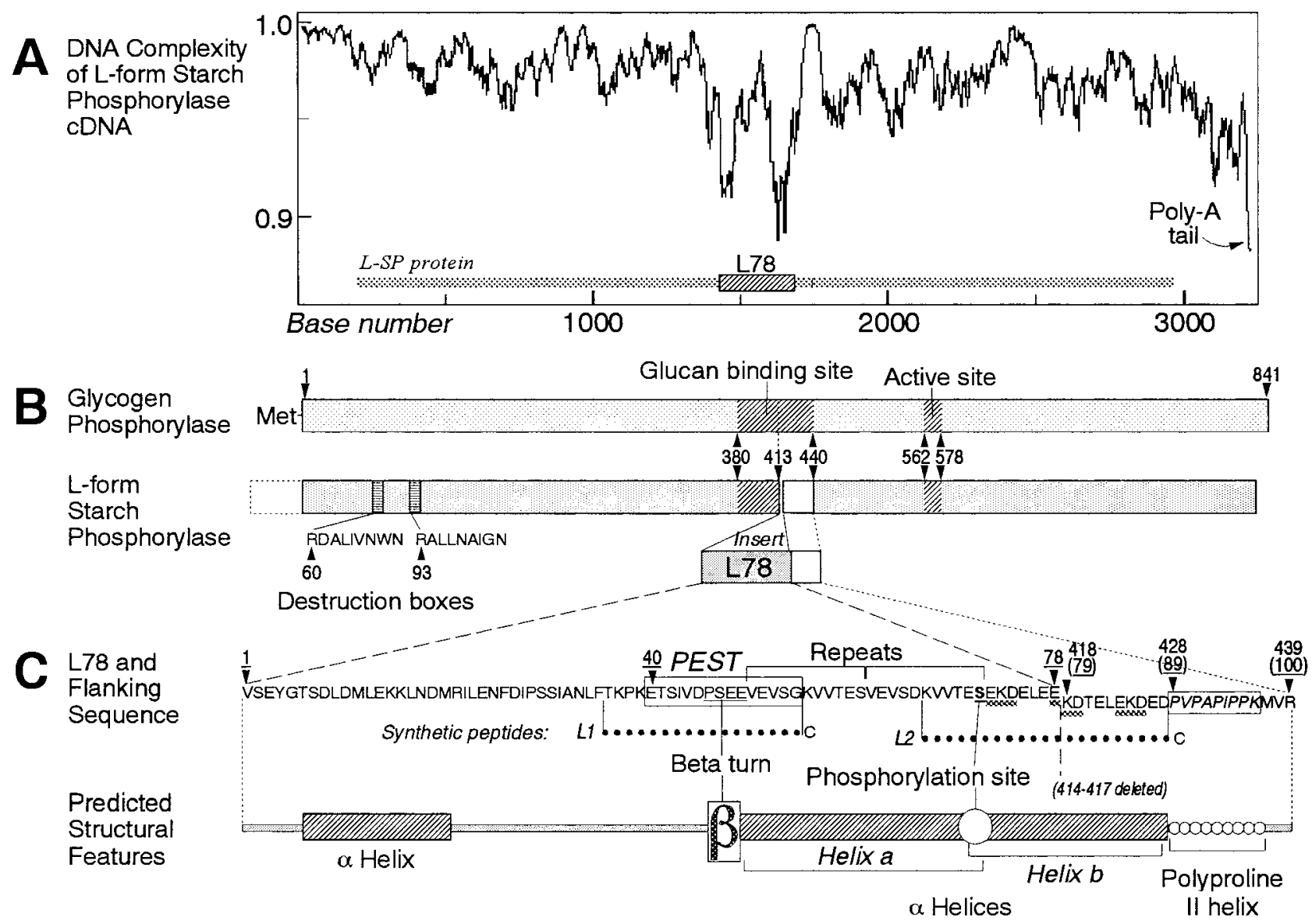

Fig. 1. Structural features of L-form starch phosphorylase from sweet potato roots. (A) The DNA complexity pattern of L-SP cDNA was generated by the program 'Complex' from PC/GENE (ver. 6.8, IntelliGenetics, Inc., Mountain View, CA, USA). (B) Comparison of the linear representations of glycogen phosphorylase from rabbit muscle and L-form starch phosphorylase proteins. The L78 insertion was displayed under the main L-SP frame which was cut open at the insertion site (residues 413-414). (C) The amino acid sequence of L78 and part of the C-terminal flanking fragment (22 residues) were analysed by the program 'Garnier', 'Betaturn', 'Pestfind', and 'Prosite' from PC/ GENE to predict the secondary structure, beta turn, PEST site, and sequence signatures, respectively. The two synthetic peptide L1 and L2 were denoted by dotted lines. 
(Vectastain, $1: 1500$ dilution containing streptavidin and biotinylated alkaline phosphatase in gelatin-NET, and incubated at room temperature for $30 \mathrm{~min}$ before use) was added to bind the biotinylated second antibody. After incubation at room temperature for $1 \mathrm{~h}$, the blot was washed twice by PBST, and then twice by alkaline phosphatase buffer (100 $\mathrm{m} M$ Tris, pH 9.5, containing $100 \mathrm{~m} M \mathrm{NaCl}$ and $10 \mathrm{~m} M \mathrm{MgCl}_{2}$ ). The bands were stained by adding the substrate solution of NBT (nitro blue tetrazolium) and BCIP (5-bromo-4-chloro-3-indolyl-phosphate) as described (Harlow and Lane 1988). The purple bluish colour bands developed in 5-20 min. After washing several times in distilled water, the blot was immersed in pure methanol for $1 \mathrm{~min}$, and then washed with distilled water before drying.

Native disc-PAGE gels were stained for SP activity (Chang et al. 1987). Following electrophoresis, the gel was incubated in a substrate solution (20 m $M$ MES, pH 5.9, containing $8 \mathrm{~m} M$ Glc-1-P and $0.3 \%$ soluble starch) for $2 \mathrm{~h}$ at $37^{\circ} \mathrm{C}$. The gel was washed with three changes of distilled water (50 $\mathrm{ml}$ each) and bands containing polymerized glucan were visualized by adding the iodine solution $\left(0.2 \% \mathrm{I}_{2}, 1.4 \% \mathrm{KI}\right.$ in distilled water, w/v). A darkish brown colour appeared very quickly. Finally, it was stopped by replacing the iodine solution with distilled water before the background darkened.

\section{Amino acid sequence analysis of F50 fragments}

Fragments of L-SP molecule (F50) were isolated by the preparative gel electrophoresis using $12.5 \%$ SDS-PAGE gel. Following electrophoresis, bands were transferred to Immobilon-P as described above, except that the transfer buffer was changed to $10 \mathrm{~m} M$ CAPS (2-[cyclohexylamino]-1-propanesulphonic acid, Sigma C-2632), pH 11, containing $10 \%$ methanol. The bands were visualized by staining briefly in Coomassie Brilliant Blue R (0.1\% solution in $40 \%$ methanol and $1 \%$ acetic acid), and then destained with $50 \%$ methanol. The bands identified as L-SP or F50 were cut out and sent for the automatic amino acid sequencing (ABI 477 A Protein Sequencer).

\section{Results}

\section{Structural features of L-form starch phosphorylase from sweet potato}

Examining the nucleotide sequence or its deduced amino acid sequence of sweet potato L-SP (Lin et al. 1991) revealed several structural characters. Figure 1 summarized these features in which the $\mathrm{L} 78$ sequence was analysed in detail. The computer program 'Complex' in PC/ GENE (IntelliGenetics, Inc.) calculates the local compositional complexity of a nucleic acid sequence, which can help map the positions of introns and exons in DNA sequence. Exon sequence in general has a higher complexity than intron (Konopka and Owens 1990). The DNA complexity pattern in Fig. 1A revealed two significant decreases in complexity in the middle of the L-
SP cDNA sequence. Compared to its deduced amino acid sequence, the two valleys in the cDNA sequence closely corresponded to the borders of L78. This observation is consistent with the hypothesis of Nakano and Fukui (1986) for potato L-SP, and of Brisson et al. (1989), in which L78 was suggested to have been derived from an intron.

The amino acid sequences of L-SP and GP were compared and shown in Fig. 1B. The alignment and numbering of amino acids in sweet potato L-SP followed that used by Lin et al. (1991), in which the numbering system of rabbit GP was employed. The active site of sweet potato L-SP (residues 562-578) was highly conserved when compared to rabbit GP or potato L-SP. The glycogenbinding site of GP contained around 60 amino acid residues (residues 380-440); whereas in the centre of the LSP starch-binding site, the L78 was inserted between residues 413 and 414. Sequence conservation of glucanbinding sites on these two types of phosphorylases was preserved only before the insertion site (residues 380413). The C-terminal half of the starch-binding sequence of L-SP (residues 418-439) showed a rather low similarity to the corresponding region on rabbit GP, and only moderate similarity to potato SP in this region. Four amino acids (residues 414-417) were absent in the L-SP from sweet potato. Near the N-terminus, destruction boxes were found in L-SP from potato and sweet potato (Fig. 1B). These boxes consist of a short stretch of conserved amino acids (RXXLXXXXN) which was found in proteins degraded by ubiquitin-dependent proteolysis (Glotzer et al. 1991). H-SP from potato contained only one, whereas rabbit GP had no functional destruction box.

The amino acid sequence of L78 was aligned and numbered from 1 to 78 using underlined numbers (Fig. 1C). A fragment of 100 amino acids containing the L78 and its C-terminal flanking sequence (22 amino acids, ranging from 418 to 439 ) were analysed to predict its secondary structure, signatures, and possible phosphorylation sites using programs in PC/GENE. A beta turn (PSEE) was predicted in the middle of the L78, suggested that L78 might form the shape of a 'loop' on the surface of the SP molecule as proposed by Nakano and Fukui (1986). A PEST sequence was found overlaying the beta turn (Glu $\underline{40}$ to Gly $\underline{54}$ ). PEST regions vary from 12 to 60 residues in length containing one or more regions rich in proline, glutamic acid, serine and threonine. Many proteins that contain PEST regions are suspected of being rapidly degraded (Rogers et al. 1986). Two repeats of nearly identical sequence (VEVSXKVVTES) followed the beta turn. They were predicted to form an alpha helix (Helix $a$, from Val $\underline{50}$ to Ser $\underline{71}$ ). The helical structure continued, and was designated as Helix $b$ (Ser 71 to Asp 88 or 427). The Ser 71 on the junction of these two helices was predicted as a strong phosphorylation site for protein kinase $\mathrm{C}$ and casein kinase II. To the C-terminal side of Helix $b$, a proline-rich segment (PVPAPIPPK) existed which might shape a left-handed polyproline II helix. Some of the polyproline II helices bound 
A SDS-PAGE:

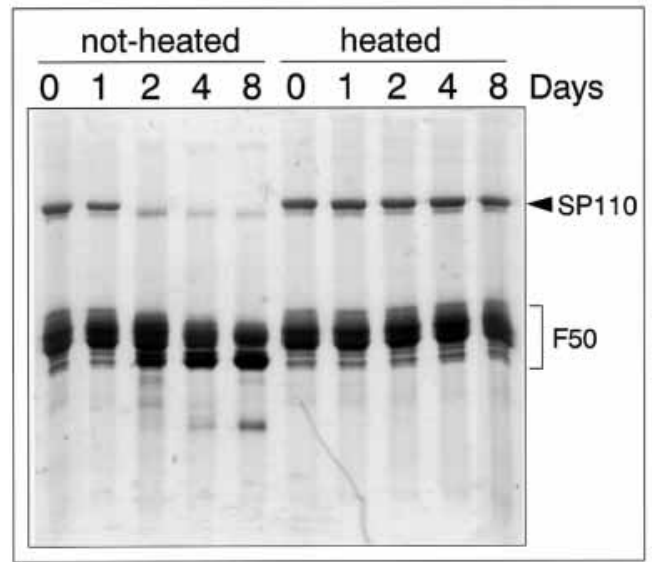

B Disc-PAGE:

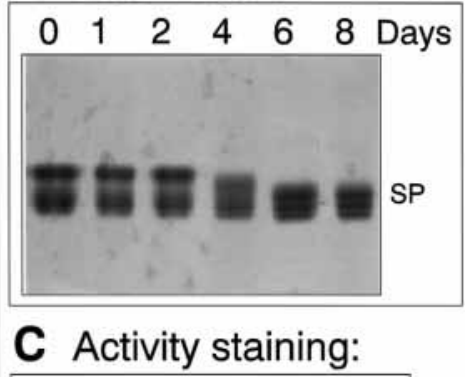

$\begin{array}{llllll}0 & 2 & 4 & 6 & 8 & \text { Days }\end{array}$

- 100
Fig. 2. Electrophoretic analysis of the proteolytic modification of sweet potato LSP. Starch phosphorylase was incubated at $37^{\circ} \mathrm{C}$ for $0,1,2,4,6$ or 8 days before electrophoresis. (A) SDS-PAGE analysis of the L-SP samples which were stained by ammonical silver. The SP110 band disappeared as the incubation period proceeded. No degradation was observed if the samples were heated (boiled for 10 min) before incubation. (B) Disc-PAGE analysis of the samples, and bands are visualized by ammoniacal silver staining. (C) The disc-PAGE gel was also stained for the starch-synthesizing activity of LSP. Apparently all samples showed amylose-forming activity on the gel.

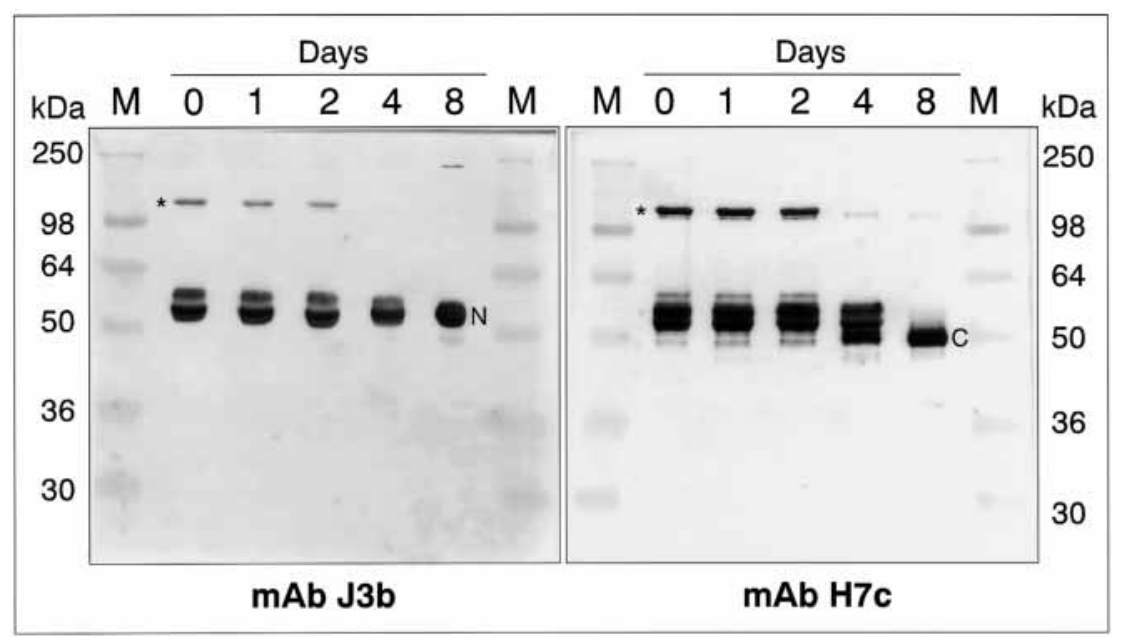

Fig. 3. Monoclonal antibodies J3b and $\mathrm{H} 7 \mathrm{c}$ were used to detect L-SP and its proteolytic fragments on SDS-PAGE $(12.5 \%)$ blots. Starch phosphorylase was incubated at $37^{\circ} \mathrm{C}$ for $0,1,2,4$, or 8 days before electrophoresis. Pre-stained markers (M) were used in both blots. Letters $\mathrm{N}$ and $\mathrm{C}$ denoted the final stable peptides after the proteolytic modification. SP110 bands were marked with asterisks $(*)$.
SH3 domains in the signal transduction pathway (Cohen et al. 1995). In this study, we have synthesized two peptides (L1 and L2 in Fig. 1C) for the production of monospecific antibodies. L1 covered the beta turn and the PEST site, and L2 contained the Ser 71 and the whole Helix $b$.

\section{Proteolytic modification of L-form starch phosphorylase}

L-SP molecules from sweet potato roots were nicked during the isolation procedures (Chern et al. 1990, Chiang et al. 1991). SDS-PAGE analysis (Day 0 in Fig. 2A) revealed that our L-SP preparation consisted of a group of fragments essentially having the molecular mass around $50 \mathrm{kDa}$ (e.g. F50). Only a small fraction of the L-SP molecules remained in their intact $110 \mathrm{kDa}$ form (e.g. SP 110). When these L-SP samples were incubated in $37^{\circ} \mathrm{C}$ for 8 days, SP110 bands continued to degrade and eventually disappeared. The mobility of F50 slightly increased, and some lower mol wt bands gradually appeared after the second day. These observations suggested that a cluster of proteolytic sites was located in the middle of the L-SP molecule or, more precisely, within L78. On the other hand, the SP110 or F50 bands did not significantly change if L-SP was heat-treated (boiled for $10 \mathrm{~min}$ ) before the incubation began. Therefore, the degradation might be caused by unknown proteolytic activity in our L-SP preparation. These proteolytic activities were not the result of contamination by microorganism, since all samples were subjected to ultrafiltration (Millex-GS, $0.22 \mu \mathrm{m}$, Millipore) before the incubation. These samples were also subjected to native disc-PAGE, stained by ammoniacal silver (Fig. 2B), or by activity staining in the direction of starch synthesis (Fig. 2C). During the incubation period, the native form L-SP changed moderately (Fig. 2B), and all samples retained their starch-synthesizing activity (Fig. 2C). Since little L-SP molecule maintained its SP110 form intact throughout this period, we concluded that the native structure of L-SP was preserved and so was its catalytic activity, despite the fact that it had been nicked in the middle of the molecule.

This study also used monoclonal antibodies ( $\mathrm{J} 3 \mathrm{~b}$ and $\mathrm{H} 7 \mathrm{c}$, see Materials and methods) to monitor the modi- 


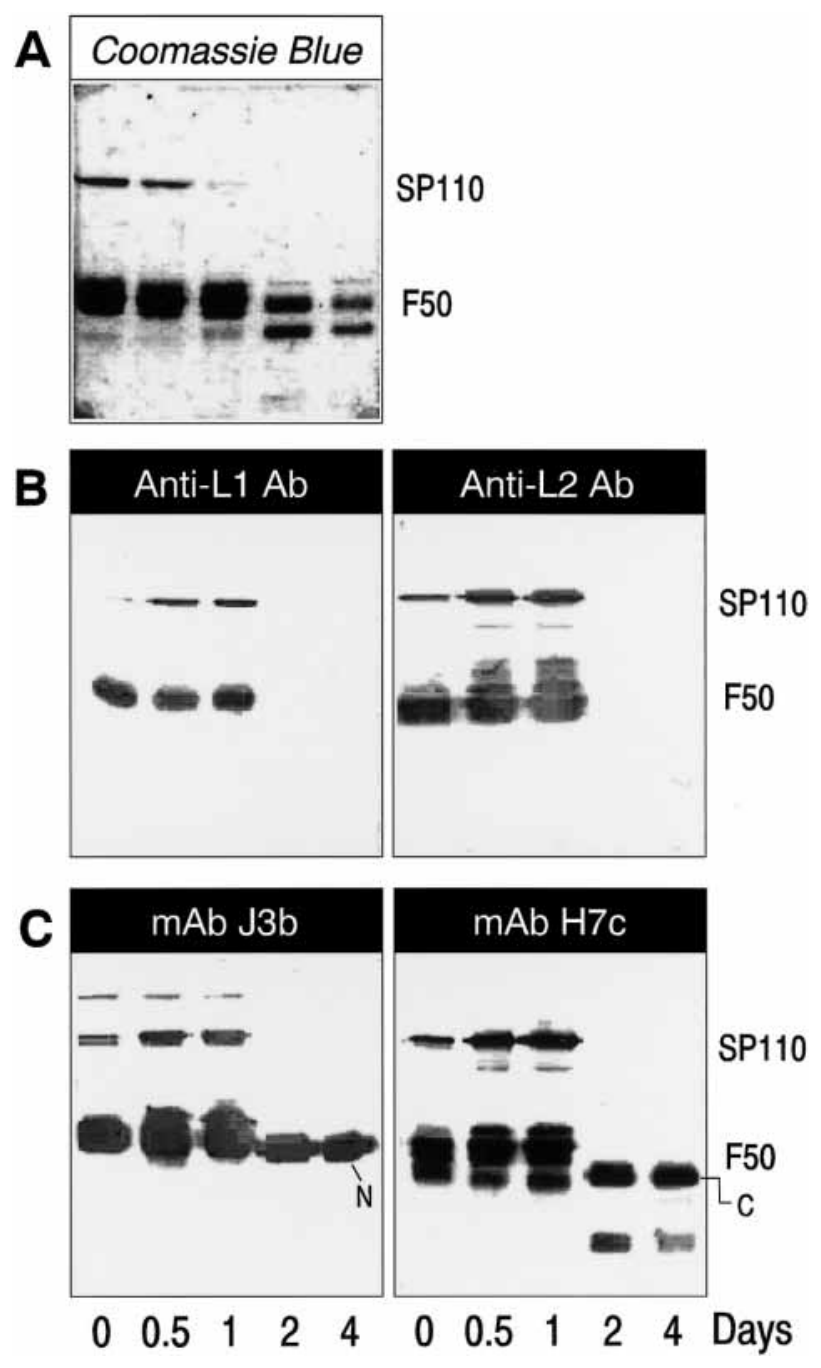

Fig. 4. The proteolytic modification process was reconstituted by adding crude extract $(2.5 \mu \mathrm{g}$ total protein) from sweet potato roots to $2.5 \mu \mathrm{g} \mathrm{L-SP}$ for $0.5,1,2$, or 4 days. (A) The degradation process was revealed by Coomassie Brilliant Blue staining. (B) Immunostaining of the SDS-PAGE blot by monospecific antibodies anti-L1 and anti-L2. (C) Immunostaining by $\mathrm{mAb} \mathrm{J} 3 \mathrm{~b}$ and $\mathrm{H} 7 \mathrm{c}$. Letters $\mathrm{N}$ and $\mathrm{C}$ denoted the two final stable peptides, respectively.

fication process (Fig.3). These two antibodies showed distinctive binding patterns on equivalent protein blots. The J3b bound to the SP110 and two F50 bands at day zero. The lower F50 band (denoted with a letter N) was resistant to further degradation throughout the incubation period, and was later identified as the N-terminal half of the SP (N436, see next section and Fig. 5). H7c bound to more F50 bands than J3b did. These F50 fragments were also degraded gradually to a stable band (denoted with a letter $\mathrm{C}$ ). This was later identified as a fragment in the C-terminal side (C416 on Fig. 5). Both monoclonal antibody staining patterns revealed that the degradation began on the second day, and lasted until the eighth day of the incubation. Detection using monoclonal antibodies simplified the protein patterns after the electrophoresis, making it possible to identify the degra- dation and elucidate the proteolytic process of SP (see Fig. 5).

Fig. 4 showed that adding a crude extract from sweet potato roots to L-SP could accelerate the degradation process. Monospecific antibodies anti-L1 and anti-L2 were used to probe specific sequences on L78 (Fig. 1C) during this process. These antibodies did not detect any band after the second day (Fig. 4B), while mAb J3b and $\mathrm{H} 7 \mathrm{c}$ bound to the stable F50 peptides ( $\mathrm{N}$ and $\mathrm{C}$ in Fig. 4C). This observation suggested that the whole C-terminal half of L78 had been excised, and the epitopes of $\mathrm{J} 3 \mathrm{~b}$ and $\mathrm{H} 7 \mathrm{c}$ were apparently not located on the excised area.

\section{Proteolytic process of L-form starch phosphorylase}

In addition to the immunostaining analysis of the F50 patterns as mentioned earlier (Fig. 3 and Fig. 4), stable F50 fragments isolated from SDS-PAGE were subjected to amino acid sequencing. The sequences obtained were aligned in Fig. 5 and denoted with asterisks (*). When compared to the amino acid sequence of L-SP from sweet potato roots (Lin et al. 1991), a process for the proteolysis of SP was proposed and illustrated. The initial translational product of L-SP mRNA consisted of 955 amino acids (Fig. 5A), in which 45 amino acids at the N-terminus were cut off and produced the $110 \mathrm{kDa}$ molecule (SP110 in Fig. 5B). SP110 was subjected to three possible major cuttings (X, Y and Z) as indicated with a pair of scissors in Fig. 5D. Major cutting site $X$ trimmed the first 14 amino acids from the $\mathrm{N}$-terminus of SP110, and produced SP108 with a new N-terminus Glu 15 (Fig. 5C). Major cutting site $\mathrm{Y}$ was the predicted PEST site on L78. This cutting produced two F50 fragments, N436 and C460, as shown in Fig. 5F (where N or $\mathrm{C}$ denoted the $\mathrm{N}$ - or $\mathrm{C}$-terminal fragments, and the following numbers represented the amino acid residues of the fragments). Major cutting site $\mathrm{Z}$ cut between Leu 422 and Glu 423, and produced fragments N480 and C416 (Fig. 5E). Both N436 and C416 were not susceptible to further degradation as described in Fig. 3. Therefore, we can isolate these stable peptides and determine the partial N-terminal amino acid sequences.

Monoclonal antibody $\mathrm{J} 3 \mathrm{~b}$ bound to an epitope on N436, and MAb H7c on C416. The two antibody symbols in Fig. 5G schematically depicted this fact; they were not used to indicate the exact epitope sites of these two antibodies. However the epitopes of the two monospecific antibodies against synthetic peptides L1 and L2 could be located precisely. Selected lanes from Fig. 3 were shown for comparison in which bands were denoted by their designated names. On the H7c pattern, an additional band (assigned as C438) clearly appeared and was sustained for several days before its disappearance. Its molecular mass was estimated by comparing to the two neighbouring bands, $\mathrm{C} 460$ and $\mathrm{C} 416$. Band C438 gradually disappeared, probably degrading to the stable C416 fragment. Therefore, at least one minor cutting site might exist in the C-terminal half of L78 (de- 
noted as L78-C in Fig. 5E,F). C460 was trimmed randomly at these minor cutting sites and produced fragments with various lengths, thereby making the $\mathrm{H} 7 \mathrm{c}$ staining pattern complex (Fig. 3). Nevertheless, N480 contained the major cutting site $\mathrm{Y}$ in addition to the L78-C and, hence, the cutting happened exclusively at site $\mathrm{Y}$ and produced the stable N436 peptide. This made the J3b pattern look simpler than H7c. These observations also implied that the major cutting site $\mathrm{Y}$ was a stronger proteolytic signal than site $\mathrm{Z}$.

\section{Higher starch affinity of modified L-form starch phosphorylase}

The enzyme kinetic studies demonstrated how this proteolytic modification affected the $\mathrm{K}_{\mathrm{m}}$ and the $\mathrm{V}_{\text {max }}$ of $\mathrm{L}$ $\mathrm{SP}$ in the direction of starch synthesis. Double reciprocal plots of L-SP during the incubation period using soluble starch as the limiting substrate were determined (Fig. $6 \mathrm{~A}$ ). The slopes of the plots shifted decreasingly (from 0 days to 8 days), and all plots intersected at a point. The $1 / \mathrm{K}_{\mathrm{m}}$ values were increasing (inset in Fig. $6 \mathrm{~A}$ ), indicating that the affinity for L-SP to starch increased during the proteolytic process. Also, the $1 / \mathrm{V}_{\max }$ values gradually decreased, reflecting an increase in its maximum velocity. On the other hand, if the Glc-1-P was used as the limiting factor, these plots change dramatically to near-parallel lines (Fig. 6B). Under this circumstance, although L$\mathrm{SP}$ also showed an increasing maximum velocity, its affinity to Glc-1-P decreased (inset in Fig. 6B) during the proteolytic process. Only a very small fraction of the L$\mathrm{SP}$ molecules remained in an intact form (SP110) at day zero in this experiment (Fig. 2A). Notably, these small number of L-SP molecules were subjected to nicking, and contributed to the changes of the $\mathrm{K}_{\mathrm{m}}$ and the $\mathrm{V}_{\max }$ as observed in this kinetic study. However, the possibility also arose that the nicked L-SP molecules were susceptible to further proteolytic modifications, thereby influencing the kinetic parameters.

Although the L-SP samples were highly purified, it was possible that these kinetic observations might be artifacts caused by trace impurity with starch-hydrolysing activity, which was unstable in $37^{\circ} \mathrm{C}$ during the incubation period. To attain direct evidence, modified L-SP



Fig. 5. A model for the proteolytic process of L-SP molecule. The proposed proteolytic cutting sites were denoted by pairs of scissors (D and F). The possible cutting fragments were shown by blocks and named according to their estimated length ('aa' denoted amino acid residues). Molecular mass in parentheses was calculated from the amino acid sequence of the fragment. Two final stable peptides (N436 and $\mathrm{C} 416)$ were obtained after exhausted proteolytic procedure $(\mathrm{G})$. The electrophoretic patterns of these peptides were simplified by using $\mathrm{mAb}$ $\mathrm{J} 3 \mathrm{~b}$ or $\mathrm{H} 7 \mathrm{c}$ (selected lanes from Fig. 3 were showed for comparison). Asterisks $(*)$ denoted the amino acid sequences which were determined by isolating the peptides and subjected to the automatic sequencing. The synthetic peptides were marked with L1 and L2 which were located mostly between the two major cutting sites $\mathrm{Y}$ and $\mathrm{Z}$. 

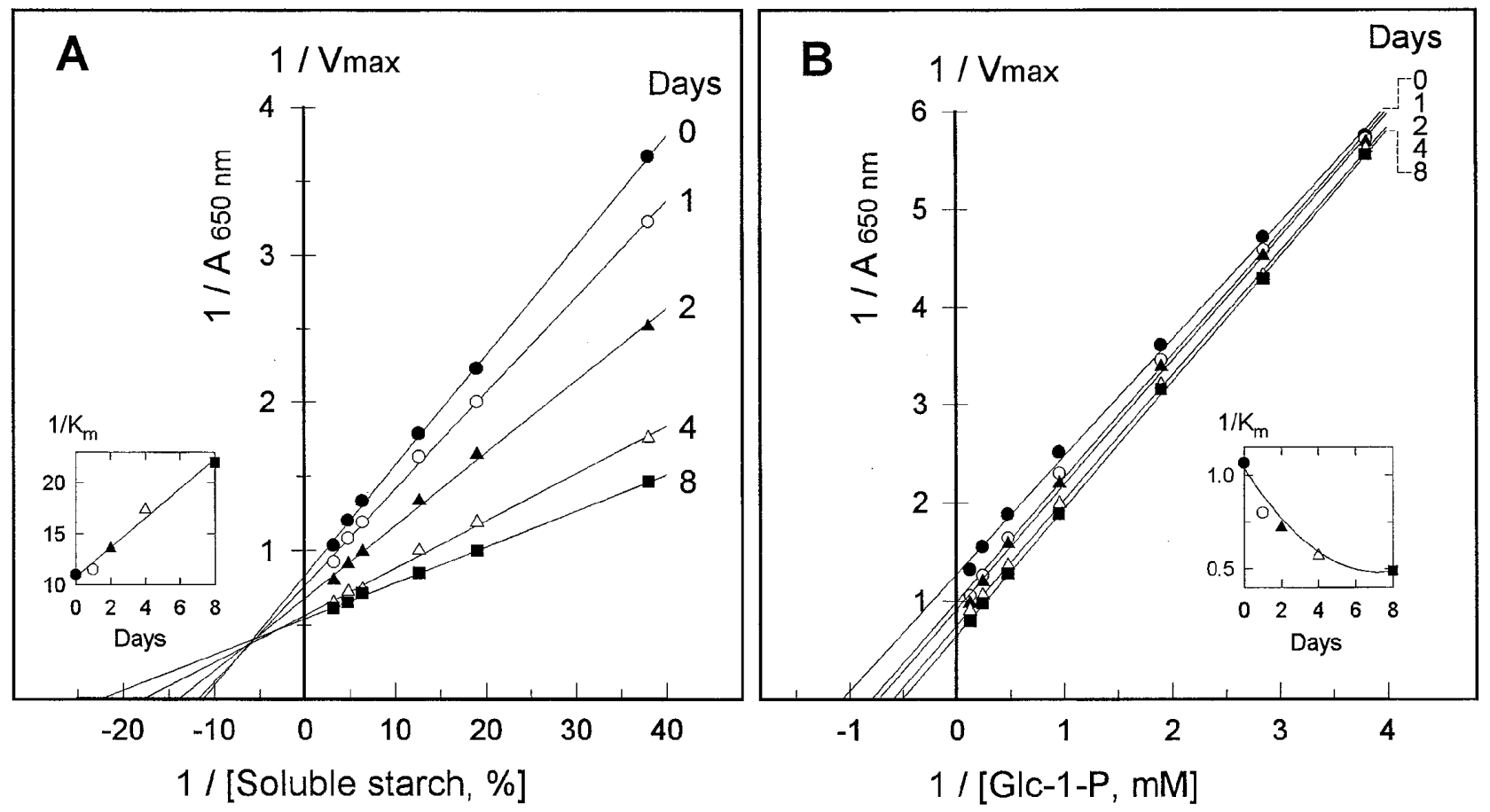

Fig. 6. Enzyme kinetic studies of the proteolytic modified L-SP molecules. L-SP was subjected to proteolysis by incubating at $37^{\circ} \mathrm{C}$ for 0 , 1 , 2,4 and 8 days before the kinetic analysis. (A) The double reciprocal plot of L-SP samples using soluble starch as the limiting substrate. (B) The plot using Glc-1-P as the limiting substrate. Insets revealed the change of $1 / \mathrm{K}_{\mathrm{m}}$ that reflected the increase or decrease of the affinity of L-SP to its substrates.

samples were subjected to disc-PAGE containing soluble starch. Figure 7 (right panel) indicated that the relative electrophoretic mobility of the modified L-SP (at 12 days or 16 days) decreased compared with the corresponding L-SP bands on day zero. Otherwise, it was comparable to the patterns of L-SP on the starch-free gel (left panel). The above results implied that the modified L-SP had a higher affinity toward starch.

\section{Discussion}

Starch phosphorylases from potato or sweet potato undergo proteolytic degradation in vitro. Brisson et al. (1989) indicated that the proteolysis of potato SP occurs in vivo and is not an artefact of the isolation procedure. According to our results, the proteolytic processing of L$\mathrm{SP}$ in sweet potato roots is heat sensitive (Fig. 2A). Reconstitute experiments (Fig. 4) also reveal that sweet potato roots contain protease activities to degrade starch phosphorylase. Interestingly, after the proteolysis, the final stable peptides (N436 and C416) have their N-terminal sequences started with Glu-Lys (Fig. 5). Therefore, we speculate that a site-specific protease might exist in sweet potato roots which recognize L-SP specifically on the sequence of X-Glu-Y (where Y is Lys in some cases). L-SP molecule is thus destined to the proteolytic process since there are four X-Glu-Lys sequences and a total of $17 \mathrm{Glu}$ residues on the $\mathrm{L} 78$ and its flanking sequence.

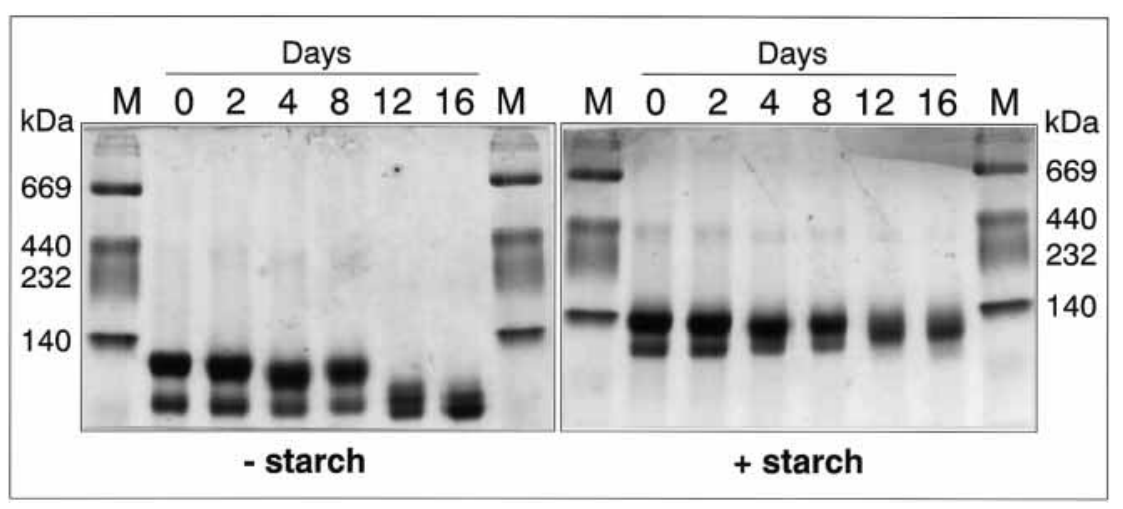

Fig. 7. Electrophoretic mobility of the proteolytic modified L-SP was retarded on the starch-containing native gel $(1.2 \%$, right panel). The molecular weight markers (M) shown on the gels served only as a reference as to how the starch influences the electrophoretic mobility of general proteins, and also as a guide for comparing the mobility of L-SP molecules. It was not intended to be used for molecular weight calibration. Although the L-SP samples were highly purified, several bands were observed on the native gel, which were caused by proteolytic modifications. 
A major cutting site $\mathrm{X}$ might be attacked by this postulated site-specific protease, which trims the 14 amino acids from the N-terminus of SP and produces a new Nterminus begun with Glu-Lys (Fig. 5C). Notably, glycogen phosphorylase is phosphorylated at Ser 14, and the phosphorylated residue causes a change of the conformation from the tense to the relaxed form (Johnson and Barford 1994). Nakano et al. (1980) reported that the potato and the rabbit muscle phosphorylases are highly homologous except the $\mathrm{N}$-terminal 33-residue region, which might provide a basis for the difference of the regulatory properties between the two enzymes. The trimming of the 14 amino acids from the N-terminus might change the conformation of L-SP, probably in a different mechanism, thereby altering the catalytic activity or the kinetic behaviour. Evidence for this pathway is still missing; nevertheless, characterization and isolation for this site-specific protease are forthcoming.

Previous studies have elucidated the mechanism for the biosynthesis of the plant starch granule to some extent (Ball et al. 1996). Related investigations focused mainly on elongation, branching and de-branching of the glucan chains, and the enzymes involved, as reviewed by Smith et al. (1997). The physiological role of starch phosphorylase in the cell has remained relatively unknown for decades. In a review article, Nelson and Pan (1995) posed the following question: 'Is there a role for phosphorylase in starch synthesis?' Tsai et al. (1970) indicated that starch phosphorylase activity increases proportionately to the starch accumulation. Molecular genetic studies also suggest that conditions favouring starch synthesis lead to an increased expression of the phosphorylase gene (St-Pierre and Brisson 1995). Besides the pioneering work of Hanes (1940), researchers have proposed that in addition to initiating primer synthesis by a glucoprotein acceptor (Tandecarz and Cardini 1978, Tandecarz and Cardini 1979), starch phosphorylase can also lengthen the glucan in the absence of an exogenous primer (Sivak et al. 1981).

$\mathrm{H}-\mathrm{SP}$ is the high-affinity isoform of phosphorylase, which lacks the L78 insertion and, hence, has a greater similarity to glycogen phosphorylase (Mori et al. 1993a). The H-SP content in sweet potato roots is much lower than the L-SP (C.Y. Chen 1995. Thesis, National Taiwan University, Taipei, Taiwan). Why do plants prefer the Lform to the H-form SP in their starch-accumulating organs? Since light is a possible inducer for the phosphorylase gene (St-Pierre and Brisson 1995), the autotrophic feature in the plant cell might be closely related. It has been proposed that phosphorylases existed originally as a large catalytically active molecule, and that the regulatory mechanism was then formed within the molecule during evolution (Nakano et al. 1980). The L78 was inserted in the starch-binding site of the ancestor phosphorylase gene during the evolution, and is sustained. This might alter the catalytic behaviour of phosphorylase from a heterotrophic catabolic role to the autotrophic biosynthesis of the starch. In this study, we focus on the L78 of L-SP, which might answer the above question.
It has been demonstrated that although L78 is subjected to extensive proteolytic modifications, the L-SP still maintains its native molecular structure and catalytic activity (Fig. 2). Other investigations have obtained similar results for the starch phosphorylase from the potato tubers (Iwata and Fukui 1973, Brisson et al. 1989). Using monospecific antibodies against specific peptides located on the L78 makes it obvious that the C-terminal half of L78 is excised out of the L-SP molecule. In addition, the stable fragments N436 and C416 are resistant to further degradation (Fig. 4). Our results have also demonstrated that the DNA complexity around the beginning and the end of L78 decreases markedly (Fig. 1A), supporting the suggestion that the L78 fragment might be derived from an intron ancestor. An interesting question arose as to why L78 was brought to be expressed through the evolution, and then designed to be excised from the L-SP molecule.

A possible reason is that the $\mathrm{L} 78$ might serve as a means of regulating L-SP by limiting the affinity of the enzyme for starch as suggested by other reports (Nakano and Fukui 1986, Brisson et al. 1989). According to our results, the L-SP $\mathrm{K}_{\mathrm{m}}$ for starch decreases in the direction of starch synthesis when the proteolytic modification proceeds (Fig.6A). Indeed, the electrophoretic mobility of the modified L-SP molecules is retarded on the gel containing soluble starch (Fig. 7). Meanwhile the affinity of L-SP for Glc-1-P is decreased (Fig. 6B). Such a decrease might be attributed to the steric hindrance of the bound starch molecule to the accessibility of Glc-1$\mathrm{P}$ toward the catalytic site of L-SP. So the intactness of L78 keeps the enzyme in a state having a higher affinity for Glc-1-P, thereby hindering the binding of starch with L-SP at the same time. Does this observation imply that L78 can facilitate the direction of the catalytic reaction of L-SP? When L78 maintains its structure intact, the catalysis by L-SP has a preference for the direction of starch synthesis using Glc-1-P as the favoured substrate. If the L78 is nicked or excised out, the enzyme binds to the starch readily and favours the direction of starch phosphorolysis. SP has been reported to catalyse the synthesis of amylose in the absence of a primer (Sivak et al. 1981). It is tempting to postulate that the L78 loop might serve as a switch that initiates the synthesis of amylose for the primer in starch biosynthesis or, on the other hand, switch to catalyse the phosphorolysis for the degradation of starch. Observations in this study suggest a possible role for the low-affinity type starch phosphorylase, and have no intention of infringing the current view of starch biosynthesis in plants.

Acknowledgements - This work was supported by National Science Council, ROC.

\section{References}

Ball S, Guan HP, James M, Myers A, Keeling P, Mouille G, Buleon A, Colonna P, Preiss J (1996) From glycogen to amylopectin - 
a model for the biogenesis of the plant starch granule. Cell 86 : 349-352

Bradford MM (1976) A rapid and sensitive method for the quantitation of microgram quantities of protein utilizing the principle of protein-dye binding. Anal Biochem 72: 248-254

Brisson N, Giroux H, Zollinger M, Camirand A, Simard C (1989) Maturation and subcellular compartmentation of potato starch phosphorylase. Plant Cell 1: 559-566

Camirand A, St-Pierre B, Marineau C, Brisson N (1990) Occurrence of a copia-like transposable element in one of the introns of the potato starch phosphorylase gene. Mol General Genet 224: 33-39

Chang TC, Lee SC, Su JC (1987) Sweet potato starch phosphorylase - purification and characterization. Agric Biol Chem 51: 187-195

Chern MS, Mo YC, Juang RH, Su JC (1990) Probing the protein structure of sweet potato starch phosphorylase with monoclonal antibodies. J Chin Biochem Soc 19: 55-64

Chiang CL, Lu YL, Juang RH, Lee PD, Su JC (1991) Native and degraded forms of sweet potato starch phosphorylase. Agric Biol Chem 55: 641-646

Cohen GB, Ren RB, Baltimore D (1995) Modular binding domains in signal transduction proteins. Cell 80: 237-248

Fiske CH, Subbarow Y (1925) The colorimetric determination of phosphorous. J Biol Chem 66: 375-400

Glotzer M, Murray AW, Kirschner MW (1991) Cyclin is degraded by the ubiquitin pathway. Nature 349: 132-138

Hanes CS (1940) The reversible formation of starch from glucose1-phosphate catalyzed by potato phosphorylase. Proc R Soc (London) B129: 174-208

Harlow E, Lane D (1988) Antibodies, a Laboratory Manual. Cold Spring Harbor Laboratory Press, New York, pp 471-510

Iwata S, Fukui T (1973) The subunit structure of $\alpha$-glucan phosphorylase from potato. FEBS Lett 36: 222-226

Johnson LN, Barford D (1990) Glycogen phosphorylase. The structural basis of the allosteric response and comparison with other allosteric proteins. J Biol Chem 265: 2409-2412

Johnson LN, Barford D (1994) Electrostatic effects in the control of glycogen phosphorylase by phosphorylation. Protein Sci 3: $1726-1730$

Juang RH, Su JC (1988) Purification of rice grain sucrose synthetase by preparative electrophoresis. J Chin Biochem Soc 17: 4251

Konopka AK, Owens JO (1990) Complexity charts can be used to map functional domains in DNA. Gene Anal Technol Appl 7: $35-38$
Laemmli UK (1970) Cleavage of structural proteins during the assembly of the head bacteriophage T4. Nature 227: 680-685

Lin CT, Yeh KW, Lee PD, Su JC (1991) Primary structure of sweet potato starch phosphorylase deduced from its cDNA sequence. Plant Physiol 95: 1250-1253

Mori H, Tanizawa K, Fukui T (1993a) A chimeric $\alpha$-glucan phosphorylase of plant type $\mathrm{L}$ and $\mathrm{H}$ isozymes: functional role of 78residue insertion in type $\mathrm{L}$ isozyme. J Biol Chem 268: 5574-5581

Mori H, Tanizawa K, Fukui T (1993b) Engineered plant phosphorylase showing extraordinarily high affinity for various $\alpha$ glucan molecules. Protein Sci 2: 1621-1629

Nakano K, Fukui T (1986) The complete amino acid sequence of potato $\alpha$-glucan phosphorylase. J Biol Chem 261: 8230-8236

Nakano K, Fukui T, Matsubara H (1980) Structural basis for the difference of the regulatory properties between potato and rabbit muscle phosphorylases. The $\mathrm{NH}_{2}$-terminal sequence of the potato enzyme. J Biol Chem 255: 9256-9261

Nakano K, Mori H, Fukui T (1989) Molecular cloning of cDNA encoding potato amyloplast $\alpha$-glucan phosphorylase and the structure of its transit peptide. J Biochem (Tokyo) 106: 691-695

Nelson O, Pan D (1995) Starch synthesis in maize endosperms. Annu Rev Plant Physiol Plant Mol Biol 46: 475-496

Newgard CB, Hwang PK, Fletterick RJ (1989) The family of glycogen phosphorylases: structure and function. Crit Rev Biochem Mol Biol 24: 69-99

Rogers S, Wells R, Rechsteiner M (1986) Amino acid sequences common to rapidly degraded proteins: the PEST hypothesis. Science 234: 364-368

Sivak MN, Tandecarz JS, Cardini CE (1981) Studies on potato tuber phosphorylase-catalyzed reaction in the absence of an exogenous acceptor. I. Characterization and properties of the enzyme. Arch Biochem Biophys 212: 525-536

Smith AM, Denyer K, Martin C (1997) The synthesis of the starch granule. Annu Rev Plant Physiol Plant Mol Biol 48: 65-87

St-Pierre B, Brisson N (1995) Induction of the plastidic starch-phosphorylase gene in potato storage sink tissue. Planta 195: 339-344

Tandecarz JS, Cardini CE (1978) A two-step enzymatic formation of a glucoprotein in potato tuber. Biochim Biophys Acta 543: 423-429

Tandecarz JS, Cardini CE (1979) Effect of 1,5-gluconolactone on the formation of a glucoprotein in potato tuber. Plant Sci Lett 15: $151-158$

Tsai CY, Nelson OE (1968) Phosphorylase I an II of maize endosperm. Plant Physiol 43: 103-112

Tsai CY, Salamini F, Nelson OE (1970) Enzymes of carbohydrate metabolism in the developing endosperm of maize. Plant Physiol 46: 299-306 